\title{
Selective proliferation of intestinal Barnesiella under fucosyllactose supplementation in mice
}

\author{
Gisela A. Weiss ${ }^{1,2}$, Christophe Chassard ${ }^{3}$ and Thierry Hennet ${ }^{1 *}$ \\ ${ }^{1}$ Institute of Physiology and Zurich Center for Integrative Human Physiology, University of Zurich, \\ Winterthurerstrasse 190, CH-8057, Zurich, Switzerland \\ ${ }^{2}$ Clinical Chemistry and Biochemistry, University Children's Hospital Zurich, Zurich, Switzerland \\ ${ }^{3}$ Laboratory of Food Biotechnology, Institute of Food, Nutrition and Health, ETH Zurich, Switzerland \\ (Submitted 29 May 2013 - Final revision received 14 November 2013 - Accepted 3 December 2013 - First published online 10 January 2014)
}

\section{Abstract}

The oligosaccharides 2-fucosyllactose and 3-fucosyllactose are major constituents of human breast milk but are not found in mouse milk. Milk oligosaccharides have a prebiotic action, thus affecting the colonisation of the infant intestine by microbiota. To determine the specific effect of fucosyllactose exposure on intestinal microbiota in mice, in the present study, we orally supplemented newborn mice with pure 2-fucosyllactose and 3-fucosyllactose. Exposure to 2-fucosyllactose and 3-fucosyllactose increased the levels of bacteria of the Porphyromonadaceae family in the intestinal gut, more precisely members of the genus Barnesiella as analysed by $16 \mathrm{~S}$ pyrosequencing. The ability of Barnesiella to utilise fucosyllactose as energy source was confirmed in bacterial cultures. Whereas B. intestinibominis and B. viscericola did not grow on fucose alone, they proliferated in the presence of 2-fucosyllactose and 3-fucosyllactose following the secretion of linkagespecific fucosidase enzymes that liberated lactose. The change in the composition of intestinal microbiota mediated by fucosyllactose supplementation affected the susceptibility of mice to dextran sulphate sodium-induced colitis, as indicated by increased resistance of mice subjected to 2-fucosyllactose supplementation for 6 weeks. The present study underlines the ability of specific milk oligosaccharides to change the composition of intestinal microbiota and thereby to shape an intestinal milieu resilient to inflammatory diseases.

\section{Key words: Fucosyllactose: Oligosaccharides: Breast milk: Microbiota}

Oligosaccharides are major constituents of human breast milk Milk oligosaccharides are built up by multiple glycosyltransferases expressed in the secretory mammary gland by extending the lactose core, thereby forming about 200 various structures $^{(1)}$. Milk oligosaccharides do not deliver energy to the newborn as the human gut lacks the glycosidase machinery required to break down these complex carbohydrates. Rather, milk oligosaccharides have a prebiotic action providing a nutrient milieu for the microbial colonisation of the intestine ${ }^{(2)}$, thereby influencing the development of the mucosal immune system of breast-fed infants ${ }^{(3)}$. Milk oligosaccharides shape the intestinal flora by providing fermentable carbohydrates to selected bacterial groups and by acting as soluble receptors preventing the adhesion of other bacteria ${ }^{(4-6)}$. The remarkable diversity of milk oligosaccharides hampers the attribution of specific functions to individual structures. To date, only a few studies have unravelled the biological impact of single milk oligosaccharides. For example, lacto- $N$-fucopentaose III was shown to induce the production of IL-10 and PGE2 in spleen cells ${ }^{(7)}$.
Disialyllacto- $N$-tetraose was shown to lower the incidence of necrotising enterocolitis in rats $^{(8)}$. Finally, 3-sialyllactose was shown to influence the intestinal abundance of clostridial cluster IV bacteria in mice and hence their susceptibility to dextran sulphate sodium (DSS)-induced colitis ${ }^{(9)}$.

Fucosyllactose is a major constituent of human milk oligosaccharides $^{(10)}$, whereas only trace amounts are found in mouse milk $^{(11)}$. Fucosyllactose occurs as Fuc $(\alpha 1-2) \mathrm{Gal}(\beta 1-4) \mathrm{Glc}$, also called 2-fucosyllactose (2-FL), and Fuc $(\alpha 1-3)(\mathrm{Gal}(\beta 1-4)) \mathrm{Glc}$, called 3-fucosyllactose (3-FL). 2-FL is similar to the blood group $\mathrm{H}$-antigen and 3-FL to the Lewis-X antigen found on glycoproteins and glycolipids ${ }^{(12)}$. 2-FL accounts for $20 \%$ of total human milk oligosaccharides and 3-FL for less than $1 \%{ }^{(13)}$. The abundance of 2-FL in human milk suggests an evolutionary pressure pertaining to the development of beneficial effects on infant physiology. Along the way, ex vivo and in vivo studies have shown that 2-FL inhibits the mucosal attachment and intestinal colonisation of Campylobacter jejuni, hence lowering the incidence of diarrhoea ${ }^{(14,15)}$. Fucosyllactose also acts as

Abbreviations: 2-FL, 2-fucosyllactose; 3-FL, 3-fucosyllactose; DGGE, denaturing gradient gel electrophoresis; DSS, dextran sulphate sodium; YCFA, yeast extract, casitone, fatty acid. 
a protected source of lactose for bacteria expressing fucosidase enzymes, such as bifidobacteria and Bacteroides, whereas Escherichia coli and Clostridium perfringens cannot utilise 2-FL as nutrient ${ }^{(16)}$.

The potential of specific oligosaccharides to influence intestinal colonisation by commensal microbiota in vivo is not known. To address the potential contribution of fucosyllactose to shaping of the distribution of intestinal microbiota, we supplemented newborn mice with 2-FL and 3-FL, which are naturally not found in mouse milk. The lack of fucosylated oligosaccharides in mouse milk may decrease the selection of fucosidase-expressing bacteria. The exposure of infant mice to soluble 2-FL and 3-FL may also impair the adhesion of bacteria to fucosylated glycans in the gastrointestinal mucosa or interfere with the bacterial regulation of host-derived fucosylated glycans through interaction with bacterial carbohydrate sensors, such as the FucR of Bacteroides thetaiotaomicron ${ }^{(17)}$. Previous experiments have demonstrated the effect of the milk oligosaccharide 3-sialyllactose on microbial composition as well as on the extent of chemically induced intestinal inflammation ${ }^{(9)}$. Beyond the effect on intestinal microbial colonisation, the question as to whether fucosyllactose supplementation has an impact on the severity of an inflammatory disease was addressed using the DSS-induced colitis model.

\section{Experimental methods}

\section{Fucosyllactose supplementation in mice}

Newborn C57BL/6 mice were fed orally with $500 \mathrm{mmol} / \mathrm{l}$ of 2-FL and 3-FL (Glycom) daily from day 1 to day 20 after birth starting with $5 \mu \mathrm{l}$, increasing by $2.5 \mu \mathrm{l}$ every $2-3 \mathrm{~d}$ reaching a daily amount of $25 \mu \mathrm{l}$ on day 20. Sterile water was given as negative control. For supplementation after weaning, 2-FL and 3-FL were added to drinking-water $(3.3 \mathrm{mmol} / \mathrm{l})$ from day 21 to day 45. Fresh faeces were collected on day 21 and day 45 and immediately stored at $-80^{\circ} \mathrm{C}$ until further analysis.

\section{Dextran sulphate sodium-induced colitis}

From day 45 postpartum, $2 \cdot 75 \%$ (w/v) of DSS (catalogue no. 0216011090; MP Biomedicals) was added to drinking-water for $5 \mathrm{~d}$ followed by provision of normal drinking-water for $2 \mathrm{~d}$ until mice were killed. Body weight was monitored daily. Mice in which body weight decreased to $85 \%$ of the initial body weight were killed immediately. Experiments were carried out in compliance with the Swiss Animal Protection Ordinance and approved by the local veterinary authority (Kantonales Veterinäramt Zürich, Switzerland). Faecal calprotectin concentrations were determined by ELISA (Immunodiagnostik) following the manufacturer's protocol.

\section{Bacterial DNA extraction and amplification}

DNA was isolated from faecal samples using the QIAamp DNA Stool Mini Kit (catalogue no. 51 504; Qiagen) according to the manufacturer's instructions. To detect cells that are difficult to lyse, the temperature at which faecal samples were incubated in lysis buffer was increased to $95^{\circ} \mathrm{C}$. The DNA concentrations of extracts were measured using NanoDrop (Witec AG). Aliquots of $100 \mathrm{ng}$ of extracted DNA were subjected to PCR using the $16 \mathrm{~S}$ rDNA universal heteroduplex analysis (HDA) primers HDA1-GC 5'-CGCCCGGGGCGCGCCCCGGGCGGGGCGGGGGCACGGGGGGACTCCTACGGGAGGCAGCAGT-3' and HDA2 $5^{\prime}$-TTACCGCGGCTGCTGGCA- $3^{\prime}$ at $56^{\circ} \mathrm{C}$ for strand annealing. Initial denaturation at $94^{\circ} \mathrm{C}$ for 4 min was followed by thirty cycles of $30 \mathrm{~s}$ at $94^{\circ} \mathrm{C}, 30 \mathrm{~s}$ at $56^{\circ} \mathrm{C}$ and $1 \mathrm{~min}$ at $72^{\circ} \mathrm{C}$. The quality of PCR products was verified by agarose gel electrophoresis.

\section{Denaturing gradient gel electrophoresis and species identification}

Amplified 16S rDNA fragments were separated by denaturing gradient gel electrophoresis (DGGE) using an INGENYphorU (Ingeny International BV) system equipped with $6 \%$ polyacrylamide gels with a denaturant in the range of $30-55 \%$, where $100 \%$ denaturant is equivalent to $7 \mathrm{M}$-urea and $40 \%$ formamide. Electrophoresis was carried out at $130 \mathrm{~V}$ for $4.5 \mathrm{~h}$ at $60^{\circ} \mathrm{C}$. Polyacrylamide gels were stained with GelRed $^{\mathrm{TM}}$ nucleic acid stain (catalogue no. 41003 ; Biotium) for $45 \mathrm{~min}$, destained in ultrapure water and viewed under UV light. Bands of interest were excised from gels and lysed in ultrapure water. Extracted DNA was reamplified using the same primers and PCR conditions. To purify the bacterial DNA, PCR products were reloaded on a denaturant gradient gel followed by excision and lysis of selected bands. DNA samples recovered from lysed bands of the second DGGE were reamplified by PCR before purification using the QIAquick PCR Purification Kit (catalogue no. 28104; Qiagen) and sequenced (Microsynth AG). Species identification was done using the Ribosomal Microbiome Database Project Classifier tool ${ }^{(18)}$

\section{Pyrosequencing and data processing}

The V5-V6 region of 16S rRNA from faecal DNA samples was amplified using the primers 784F $5^{\prime}$-AGGATTAGATACCCTGGTA-3' and 1061R 5'-CRRCACGAGCTGACGAC-3' $3640^{(19)}$. Amplicons were pyrosequenced using a Roche 454 GS-FLX system (DNAVision). Sequences of at least 240 nucleotides and containing no more than two undetermined bases were retained for taxonomic assignment. The QIIME software ${ }^{(20)}$ was used for chimera check and the Greengenes database ${ }^{(21)}$ was used for classification. Bacterial diversity was determined at the phylum, family and genus levels. Sequences were aligned to the 16S rRNA of $B$. intestinibominis and B. viscericola with BLASTN $2.2 .27+{ }^{(22)}$. The GenBank accession numbers for the 16S rDNA sequences of $B$. intestinibominis and B. viscericola are AB267809.1 and AB370251.1, respectively.

\section{Bacterial culture}

Strains of B. viscericola and B. intestinibominis were obtained from Leibniz-Institut, Deutsche Sammlung von Mikroorganismen und Zellkulturen GmbH. Cell morphology was inspected visually by microscopy (DM1000 $10 \times / 20$ microscope; Leica). 


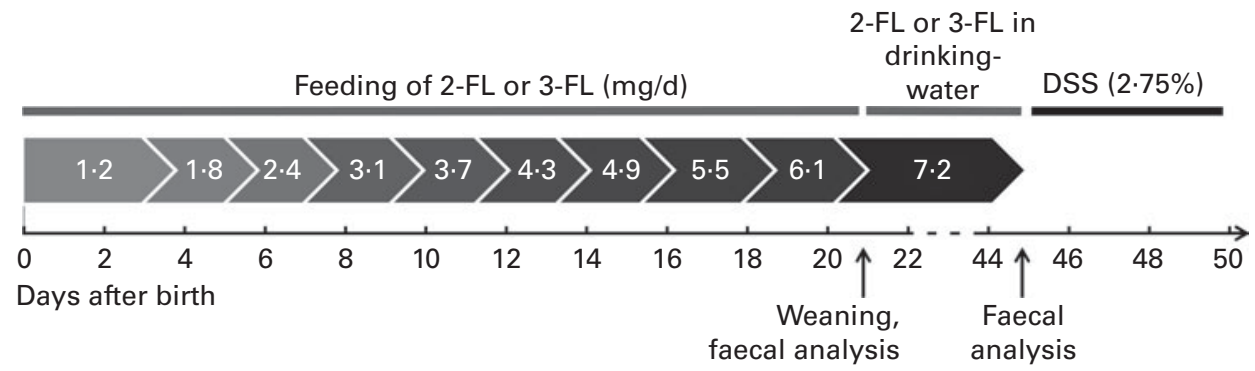

Fig. 1. Fucosyllactose (2-FL or 3-FL) supplementation protocol. Fucosyllactose was fed orally until weaning at increasing daily amounts. Fucosyllactose supplementation after weaning was administered through drinking-water. Average daily consumption of $4.5 \mathrm{ml}$ drinking-water per $\mathrm{d}$ in one mouse resulted in a daily intake of $7.2 \mathrm{mg}$ fucosyllactose. Dextran sulphate sodium (DSS, $2.75 \%$ ) was added to drinking-water from day 45 to day 50.

Substrate utilisation was determined by adding sterilised, $\mathrm{O}_{2}$-free sugar solutions of glucose, lactose, fucose, 2-FL or 3-FL at a final concentration of $2.44 \mathrm{~g} / 1$ to yeast extract, casitone, fatty acid (YCFA) medium ${ }^{(23)}$. YCFA medium was dispensed in $8 \mathrm{ml}$ aliquots in Hungate tubes sealed with butyl rubber septa. SCFA were added to the medium at the final concentrations of $32.4 \mathrm{mmol}$ acetate $/ 1, \quad 4.7 \mathrm{mmol}$ butyrate/1, $8.7 \mathrm{mmol}$ propionate/1, $1 \mathrm{mmol} n$-valeric acid/l and $1.2 \mathrm{mmol}$ isobutyric acid/l. YCFA medium was prepared and maintained anaerobically using $\mathrm{CO}_{2}$. Inoculated tubes were incubated at $37^{\circ} \mathrm{C}$. Growth was measured spectrophotometrically at $600 \mathrm{~nm}$.

\section{Statistical analysis}

Data are presented as means and standard deviations, unless otherwise indicated. Comparisons of means were made with $t$ test or one-way ANOVA using SPSS 20. $P$ values lower than 0.05 were considered significant.

\section{Results}

\section{Microbial variation after 3 and 6 weeks of supplementation}

To determine whether early exposure to exogenous fucosyllactose influenced intestinal microbial, we first compared three supplementation regimens. Mice were fed daily with 2-FL or
3-FL for 3 weeks from birth to weaning, for 3 weeks after weaning or for 6 weeks from birth (Fig. 1). The latter protocol was referred to as long-term supplementation. Before weaning, 2-FL and 3-FL were given orally in addition to the natural feeding of the pups through maternal milk. Microbiological diversity in faecal samples was first examined by DGGE of amplified bacterial $16 \mathrm{~S}$ rDNA fragments. By the time of weaning, the DGGE profiles obtained for the 2-FL- and 3-FL-supplemented mice and control mice were very similar with only a few DNA bands specifically being found in single supplementation groups. The main difference was represented by a band corresponding to bacteria of the Lachnospiraceae family observed in the 2-FL-supplemented mice (data not shown). DGGE profiles obtained from DNA samples isolated from mice at 6 weeks of age exhibited more pronounced changes as exemplified by prominent bands representing members of the Porphyromonadaceae family (Fig. 2). The increase in the bacterial numbers of the Porphyromonadaceae family was strongest in mice supplemented with 2-FL for 6 weeks, whereas mice supplemented with 2-FL and 3-FL for 3 weeks after weaning also exhibited an increase in bacterial numbers, yet to a lesser extent than mice exposed to 2-FL or 3-FL for the whole study period. The DGGE band corresponding to the bacteria of the Porphyromonadaceae family was weaker in mice supplemented with 2-FL than in those supplemented with 3-FL for 3 weeks after weaning. Because of the limited sensitivity

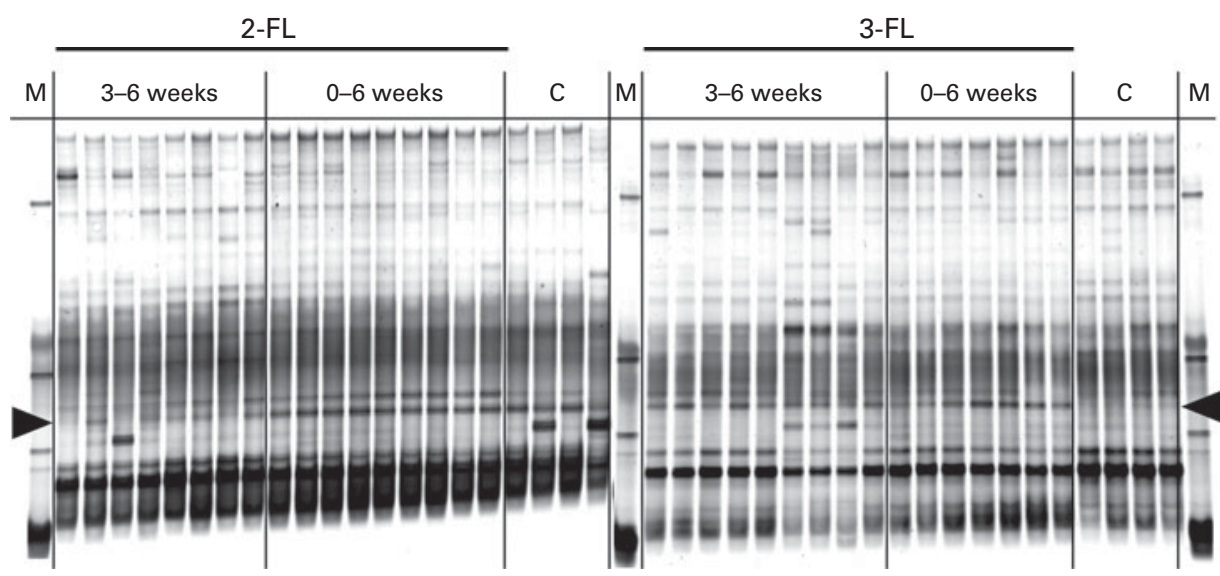

Fig. 2. Denaturing gradient gel electrophoresis profiles of bacterial $16 \mathrm{~S}$ rDNA isolated from faecal samples at the age of 6 weeks. Variations in bacterial composition in the 2-fucosyllactose (2-FL)-supplemented, 3-fucosyllactose (3-FL)-supplemented and control mice ( $n$ 8) were compared. The duration of 2-FL or 3-FL supplementation is indicated as 3-6 weeks ( $n 8$ and 9, respectively) or 0-6 weeks ( $n 9$ and 7, respectively). Each lane represents DNA of faecal bacteria from one mouse. The arrow heads at the left and right point to a band corresponding to a member of the Porphyromonadaceae family. $\mathrm{M}$, marker; $\mathrm{C}$, control. 
of DGGE to quantify microbial diversity, we also analysed the microbial composition of DNA samples using high-throughput sequencing.

Each sample was analysed at coverage ranging from 6898 to $1617116 \mathrm{~S}$ rDNA sequences. The grouping of sequences at the level of bacterial families confirmed the results obtained by DGGE. Mice supplemented with 2-FL from birth for 3 weeks had increased levels of bacteria of the Lachnospiraceae family (Fig. 3(a)), whereas those of bacteria of other families did not vary significantly after exposure of mice to 2-FL and 3-FL (Table 1). Reciprocally, the abundance of bacteria of the Alcaligenaceae family strongly decreased in the 2-FLsupplemented mice, while that of bacteria of the remaining families remained unchanged in the 3-FL-supplemented mice. The dominant bacterial families identified at 3 weeks of age were Porphyromonadaceae and Bacteroidaceae, which accounted for, respectively, 39 and $16 \%$ of sequence coverage in the control mice. After weaning at 3 weeks of age, the transition from breast milk to solid chow led to several adjustments in the distribution of intestinal microbiota in the control and fucosyllactose-supplemented mice (Table 1). The bacteria of the Porphyromonadaceae family remained dominant in all mouse groups at 6 weeks of age, yet with a sharp increase in numbers in the 2-FL- and 3-FL-supplemented mice (Fig. 3(b)). The analysis of bacterial sequences at the genus level revealed that Barnesiella accounted for the observed increase in the bacterial numbers of the Porphyromonadaceae family (Table 2). At the age of 6 weeks, varying levels of Barnesiella were observed in the three mouse groups (Fig. 3(c)). Whereas Barnesiella summed up to $58.3 \%$ of the identified genera in the 2-FL-supplemented mice, Barnesiella summed up to, respectively, $47 \cdot 0$ and $39.6 \%$ of the genera in the 3-FL-supplemented mice and control mice.

\section{Fucosyllactose utilisation by Barnesiella}

To date, two species of Barnesiella, namely $B$. viscericola isolated from chicken caecum and B. intestinihominis from human faeces, have been characterised and cultivated ${ }^{(24,25)}$. The Barnesiella sequences obtained in the present study exhibited up to $93 \%$ identity with the reference $16 \mathrm{~S}$ rDNA sequence of $B$. intestinibominis and $B$. viscericola. The enrichment of Barnesiella found in the fucosyllactosesupplemented mice suggested that these bacteria are able to utilise fucosyllactose as carbon source. To address this question, we monitored the growth of B. viscericola and B. intestinihominis in vitro in culture medium containing glucose, lactose, fucose, 2-FL or 3-FL as the sole energy source. As expected, both Barnesiella species grew in the medium containing glucose and lactose (Fig. 4(a)-(d)). By contrast, Barnesiella failed to grow in the presence of fucose, indicating that these bacteria cannot utilise this deoxyhexose as carbon source (Fig. 4(e) and (f)). The addition of either 2-FL or 3-FL to the growth medium revealed the differential utilisation of both forms of fucosyllactose by B. viscericola and B. intestinihominis. Whereas $B$. intestinibominis was able to grow in the 2-FLcontaining medium but not in the 3 -FL-containing medium (Fig. 4(g) and (i)), the reverse phenotype was observed for
B. viscericola, which grew in the presence of 3-FL but not in the presence of 2-FL (Fig. 4(h) and (j)). Noteworthily, the growth of $B$. intestinibominis in the presence of 2-FL was delayed, suggesting that the utilisation of the oligosaccharide as energy source required the expression of a putative fucosidase. During the growth experiments, the cell morphology of
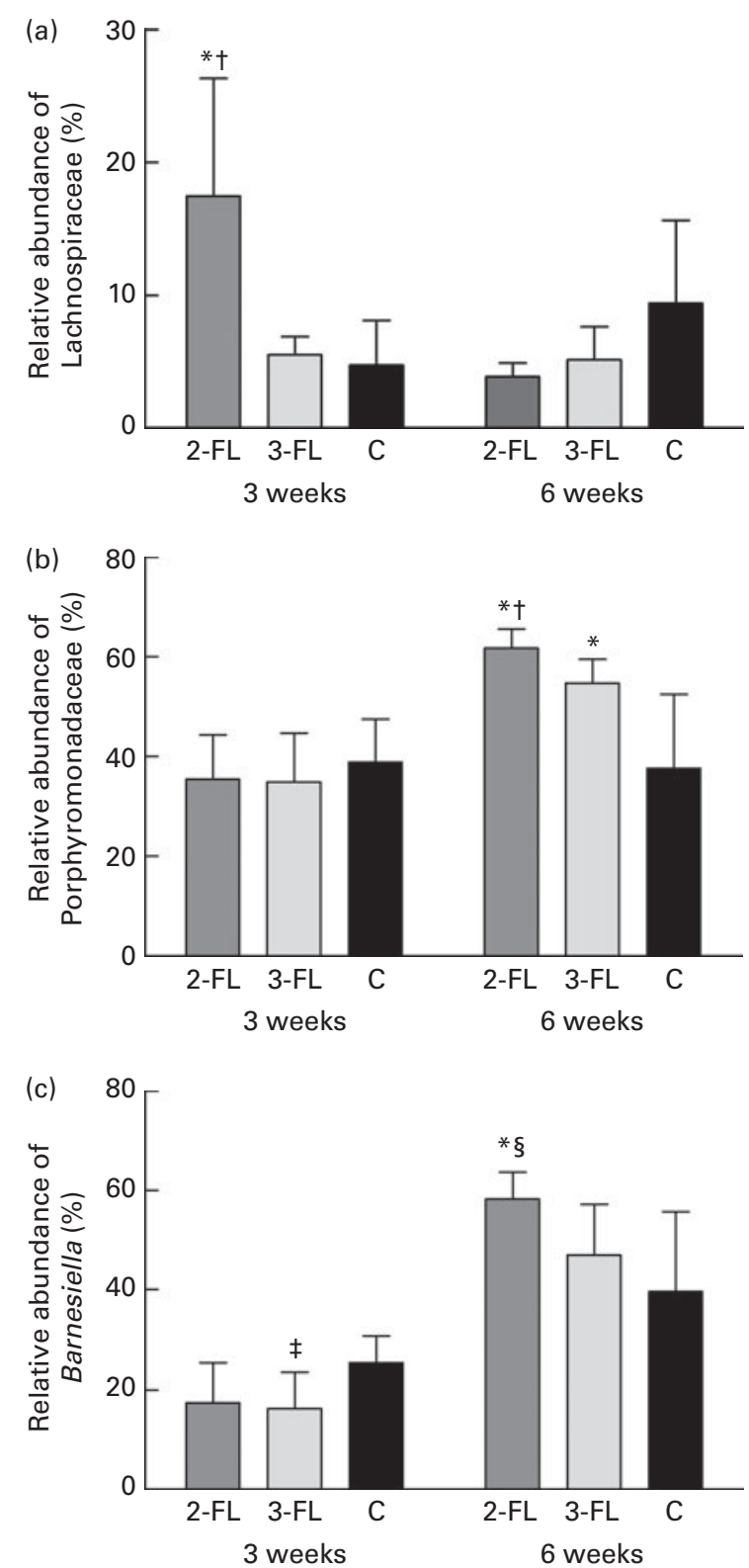

Fig. 3. Association between individual bacterial species and fucosyllactose supplementation. Relative abundances of the Lachnospiraceae family (a), the Porphyromonadaceae family (b) and the Barnesiella genus (c) were determined in the faecal samples of the long-term supplemented and control mice at 3 and 6 weeks of age by pyrosequencing. Values are means ( 5 mice per group), with standard deviations represented by vertical bars. * Mean value was significantly different from that of the control mice $(P<0.05 ; t$ test $)$. † Mean value was significantly different from that of the 3 FL-supplemented mice $(P<0.05 ; t$ test). $¥$ Mean value was significantly different from that of the control mice $(P<0.1$; trend; $t$ test). $\S$ Mean value was significantly different from that of the $3-\mathrm{FL}$-supplemented mice $(P<0.1$; trend; $t$ test). 2-FL, 2-fucosyllactose; 3-FL, 3-fucosyllactose; C, control. 
Table 1. Relative abundance of bacterial families in 2-fucosyllactose (2-FL)- or 3-fucosyllactose (3-FL)-supplemented and control mice at the age of 3 and 6 weeks

(Mean values and standard deviations; $n 5$ mice per group)*

\begin{tabular}{|c|c|c|c|c|c|c|c|c|c|c|c|c|}
\hline & \multicolumn{6}{|c|}{ After 3 weeks } & \multicolumn{6}{|c|}{ After 6 weeks } \\
\hline & \multicolumn{2}{|c|}{$2-F L$} & \multicolumn{2}{|c|}{ 3-FL } & \multicolumn{2}{|c|}{ Control } & \multicolumn{2}{|c|}{$2-\mathrm{FL}$} & \multicolumn{2}{|c|}{ 3-FL } & \multicolumn{2}{|c|}{ Control } \\
\hline & Mean & SD & Mean & SD & Mean & SD & Mean & SD & Mean & SD & Mean & SD \\
\hline Total reads $\dagger(n)$ & \multirow{2}{*}{\multicolumn{2}{|c|}{$\begin{array}{c}10377-12327 \\
37-47\end{array}$}} & \multirow{2}{*}{\multicolumn{2}{|c|}{$\begin{array}{c}6898-12181 \\
30-44\end{array}$}} & \multirow{2}{*}{\multicolumn{2}{|c|}{$\begin{array}{c}11046-16171 \\
28-48\end{array}$}} & \multirow{2}{*}{\multicolumn{2}{|c|}{$\begin{array}{c}9463-13551 \\
33-40\end{array}$}} & \multirow{2}{*}{\multicolumn{2}{|c|}{$\begin{array}{c}2624-11960 \\
30-41\end{array}$}} & \multirow{2}{*}{\multicolumn{2}{|c|}{$\begin{array}{c}3414-6888 \\
23-54\end{array}$}} \\
\hline Sequence assignment (\%) & & & & & & & & & & & & \\
\hline Alcaligenaceae & 0.5 & 0.4 & $6 \cdot 2$ & $4 \cdot 1$ & 6.9 & 9.4 & $2 \cdot 1$ & 0.5 & $2 \cdot 2$ & $2 \cdot 2$ & 1.6 & 1.0 \\
\hline Anaeroplasmataceae & 0.0 & $0 \cdot 1$ & $2 \cdot 1$ & $2 \cdot 6$ & 2.7 & 3.0 & $3 \cdot 2$ & 1.5 & 0.9 & 0.7 & 3.4 & 3.4 \\
\hline Bacteroidaceae & $27 \cdot 1$ & $13 \cdot 7$ & 24.4 & $16 \cdot 4$ & $15 \cdot 6$ & 9.5 & $5 \cdot 2$ & 1.8 & $7 \cdot 3$ & $2 \cdot 0$ & 8.4 & $10 \cdot 1$ \\
\hline Coriobacteriaceae & 0.1 & 0.1 & 0.3 & 0.2 & 0.1 & 0.2 & 0.1 & 0.0 & 0.2 & 0.1 & 0.1 & $0 \cdot 1$ \\
\hline Deferribacteraceae & 0.0 & 0.0 & 0.2 & 0.3 & 0.3 & 0.3 & 0.1 & 0.1 & 0.0 & 0.0 & 0.6 & 0.7 \\
\hline Erysipelotrichaceae & 0.1 & 0.1 & 1.8 & 1.9 & $2 \cdot 2$ & $2 \cdot 8$ & 0.7 & 0.3 & $2 \cdot 1$ & 1.2 & 0.9 & 1.3 \\
\hline Helicobacteraceae & 1.7 & $1 \cdot 1$ & $2 \cdot 1$ & 1.9 & $5 \cdot 1$ & 3.8 & $2 \cdot 7$ & 1.0 & $2 \cdot 7$ & 0.9 & $2 \cdot 4$ & $1 \cdot 2$ \\
\hline Incertae Sedis XIV & 0.2 & 0.4 & 0.1 & 0.1 & 0.0 & 0.0 & 0.0 & 0.0 & 0.0 & 0.0 & 0.0 & 0.0 \\
\hline Lachnospiraceae & $17 \cdot 4$ & 8.9 & 5.5 & 1.4 & 4.7 & 3.4 & 3.9 & 1.0 & $5 \cdot 1$ & 2.5 & $9 \cdot 4$ & $6 \cdot 2$ \\
\hline Lactobacillaceae & $2 \cdot 2$ & 1.0 & 4.7 & 4.4 & $3 \cdot 2$ & $2 \cdot 3$ & $1 \cdot 3$ & 1.5 & $1 \cdot 1$ & 1.4 & 0.6 & 0.9 \\
\hline Mycoplasmataceae & 0.7 & 0.7 & 0.2 & 0.2 & 0.7 & 0.7 & 0.4 & 0.1 & 0.5 & 0.4 & 0.1 & 0.1 \\
\hline Porphyromonadaceae & $35 \cdot 4$ & $9 \cdot 0$ & 34.8 & 9.9 & 38.8 & $8 \cdot 7$ & $61 \cdot 8$ & 3.8 & 54.8 & 4.8 & 37.6 & 14.9 \\
\hline Prevotellaceae & $5 \cdot 1$ & $3 \cdot 0$ & $3 \cdot 2$ & 1.0 & 4.4 & 3.5 & 3.7 & 0.8 & 3.7 & 0.8 & 4.9 & $3 \cdot 2$ \\
\hline Rikenellaceae & $5 \cdot 4$ & $2 \cdot 1$ & $7 \cdot 0$ & $4 \cdot 7$ & 9.4 & $5 \cdot 6$ & $6 \cdot 3$ & 0.8 & $6 \cdot 2$ & $2 \cdot 9$ & $6 \cdot 3$ & $2 \cdot 1$ \\
\hline Ruminococcaceae & $3 \cdot 8$ & 1.7 & $7 \cdot 1$ & $4 \cdot 0$ & $5 \cdot 0$ & $2 \cdot 2$ & $8 \cdot 4$ & 1.6 & $13 \cdot 1$ & 3.8 & $23 \cdot 7$ & $21 \cdot 1$ \\
\hline Verrucomicrobiaceae & 0.0 & 0.0 & 0.0 & 0.0 & 0.7 & 1.4 & 0.0 & 0.0 & 0.0 & 0.0 & 0.0 & 0.0 \\
\hline
\end{tabular}

* Only families that are present with a mean value of more than $0.2 \%$ in at least one group are displayed.

† Number of read sequences passing quality control.

Table 2. Relative abundance of bacterial genera in 2-fucosyllactose (2-FL)- or 3-fucosyllactose (3-FL)-supplemented and control mice at the age of 3 and 6 weeks

(Mean values and standard deviations; $n 5$ mice per group) ${ }^{\star}$

\begin{tabular}{|c|c|c|c|c|c|c|c|c|c|c|c|c|}
\hline & \multicolumn{6}{|c|}{ After 3 weeks } & \multicolumn{6}{|c|}{ After 6 weeks } \\
\hline & \multicolumn{2}{|c|}{$2-\mathrm{FL}$} & \multicolumn{2}{|c|}{ 3-FL } & \multicolumn{2}{|c|}{ Control } & \multicolumn{2}{|c|}{$2-\mathrm{FL}$} & \multicolumn{2}{|c|}{ 3-FL } & \multicolumn{2}{|c|}{ Control } \\
\hline & Mean & SD & Mean & SD & Mean & SD & Mean & SD & Mean & SD & Mean & SD \\
\hline Total reads $\dagger(n)$ & \multirow{2}{*}{\multicolumn{2}{|c|}{$\begin{array}{c}10377-12327 \\
21-30\end{array}$}} & \multirow{2}{*}{\multicolumn{2}{|c|}{$\begin{array}{c}6898-12181 \\
20-30\end{array}$}} & \multirow{2}{*}{\multicolumn{2}{|c|}{$\begin{array}{c}11046-16171 \\
20-38\end{array}$}} & \multirow{2}{*}{\multicolumn{2}{|c|}{$\begin{array}{c}9463-13551 \\
22-28\end{array}$}} & \multirow{2}{*}{\multicolumn{2}{|c|}{$\begin{array}{c}2624-11960 \\
20-26\end{array}$}} & \multirow{2}{*}{\multicolumn{2}{|c|}{$\begin{array}{c}3414-6888 \\
14-25\end{array}$}} \\
\hline Sequence assignment (\%) & & & & & & & & & & & & \\
\hline Akkermansia & 0.0 & 0.0 & 0.1 & 0.0 & 0.8 & $1 \cdot 7$ & 0.0 & 0.0 & 0.0 & 0.1 & 0.0 & 0.0 \\
\hline Alistipes & 8.0 & 3.7 & 8.4 & $5 \cdot 8$ & $10 \cdot 8$ & 7.5 & 8.5 & 0.9 & $7 \cdot 6$ & $3 \cdot 1$ & $10 \cdot 5$ & 1.8 \\
\hline Allobaculum & 0.0 & 0.0 & 0.6 & 0.5 & 0.0 & 0.0 & 0.7 & 0.5 & 1.6 & $1 \cdot 2$ & 0.4 & 0.8 \\
\hline Anaeroplasma & 0.1 & 0.1 & $3 \cdot 1$ & $3 \cdot 8$ & 3.6 & 4.0 & 4.7 & $2 \cdot 1$ & 1.3 & $1 \cdot 2$ & $6 \cdot 6$ & $5 \cdot 6$ \\
\hline Bacteroides & 42.5 & $20 \cdot 9$ & $34 \cdot 1$ & $21 \cdot 7$ & $20 \cdot 2$ & $11 \cdot 1$ & $7 \cdot 7$ & $2 \cdot 7$ & $11 \cdot 2$ & 3.5 & $15 \cdot 0$ & $15 \cdot 5$ \\
\hline Barnesiella & 17.4 & 8.0 & $16 \cdot 3$ & $7 \cdot 3$ & $25 \cdot 4$ & 5.4 & $58 \cdot 3$ & 5.4 & $47 \cdot 0$ & $10 \cdot 2$ & $39 \cdot 6$ & $16 \cdot 1$ \\
\hline Blautia & 0.4 & 0.7 & $0 \cdot 1$ & 0.2 & 0.0 & 0.0 & 0.0 & 0.0 & 0.0 & 0.0 & 0.0 & 0.0 \\
\hline Butyricicoccus & 0.0 & 0.0 & 0.0 & 0.0 & 0.0 & 0.0 & 0.2 & 0.2 & 0.0 & 0.1 & 0.4 & 0.6 \\
\hline Coprobacillus & 0.1 & 0.1 & 0.7 & 0.9 & 1.4 & 1.5 & 0.2 & 0.1 & 0.5 & 0.4 & 0.4 & 0.4 \\
\hline Helicobacter & $2 \cdot 5$ & 1.7 & $2 \cdot 8$ & 2.4 & $6 \cdot 1$ & 4.8 & 3.7 & 1.4 & 3.8 & 1.3 & 4.4 & 3.5 \\
\hline Lactobacillus & 3.6 & $2 \cdot 1$ & $7 \cdot 0$ & $6 \cdot 9$ & 4.4 & 3.2 & 1.9 & $2 \cdot 1$ & 1.8 & 2.4 & $1 \cdot 1$ & 1.5 \\
\hline Marvinbryantia & 0.0 & 0.0 & $0 \cdot 1$ & 0.1 & 0.0 & 0.0 & 0.1 & 0.0 & 0.1 & 0.1 & 0.3 & 0.2 \\
\hline Mucispirillum & 0.0 & 0.0 & 0.4 & 0.4 & 0.4 & 0.4 & 0.1 & 0.1 & 0.0 & 0.1 & $1 \cdot 7$ & $2 \cdot 2$ \\
\hline Odoribacter & 0.2 & 0.1 & 0.0 & 0.0 & 0.8 & 0.8 & 0.6 & 0.2 & 0.0 & 0.0 & 0.9 & 0.5 \\
\hline Oscillibacter & $2 \cdot 3$ & 1.1 & $1 \cdot 1$ & 1.0 & $1 \cdot 1$ & 0.9 & 1.8 & 0.4 & 1.9 & $1 \cdot 1$ & 3.6 & 2.6 \\
\hline Parabacteroides & 13.9 & 6.4 & $13 \cdot 1$ & 4.9 & 11.4 & 6.5 & 4.1 & $2 \cdot 2$ & 13.3 & 7.5 & $5 \cdot 0$ & $6 \cdot 4$ \\
\hline Paraprevotella & 0.0 & 0.0 & 0.0 & 0.0 & 0.3 & 0.6 & 0.0 & 0.0 & 0.0 & 0.0 & 0.0 & 0.0 \\
\hline Parasutterella & 0.4 & 0.4 & $6 \cdot 3$ & 4.2 & $7 \cdot 3$ & $10 \cdot 0$ & 1.6 & 0.3 & $2 \cdot 7$ & 2.9 & $2 \cdot 7$ & 1.6 \\
\hline Prevotella & $6 \cdot 1$ & 3.4 & $3 \cdot 6$ & $1 \cdot 1$ & 3.9 & $2 \cdot 3$ & 4.2 & 0.9 & 4.4 & 1.0 & $5 \cdot 3$ & 1.9 \\
\hline Roseburia & 0.1 & 0.1 & 0.7 & 0.6 & 0.1 & 0.2 & 0.1 & 0.1 & 0.3 & 0.2 & 0.0 & 0.1 \\
\hline TM7 genera incertae sedis & 0.4 & 0.4 & 0.5 & 0.4 & 0.1 & 0.2 & 0.5 & 0.4 & 0.8 & 0.6 & 0.4 & 0.4 \\
\hline Ureaplasma & $1 \cdot 1$ & $1 \cdot 2$ & 0.3 & 0.3 & 0.9 & 0.9 & 0.6 & 0.2 & 0.7 & 0.6 & 0.2 & 0.2 \\
\hline Xylanibacter & 0.4 & 0.2 & 0.3 & 0.3 & 0.2 & 0.1 & 0.3 & 0.2 & 0.3 & 0.1 & 0.9 & 0.7 \\
\hline
\end{tabular}

* Only genera that are present with a mean value of more than $0.2 \%$ in at least one group are displayed.

† Number of read sequences passing quality control. 
(a)

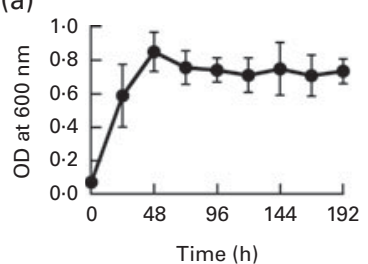

(c)

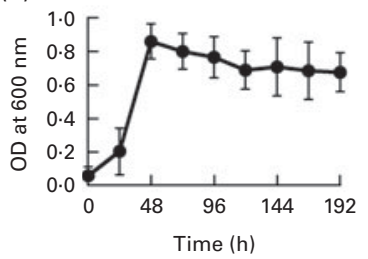

(e)

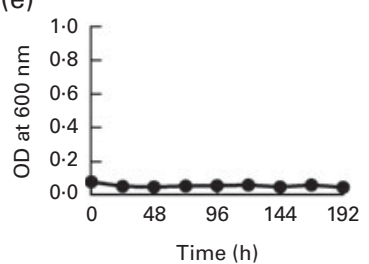

(g)

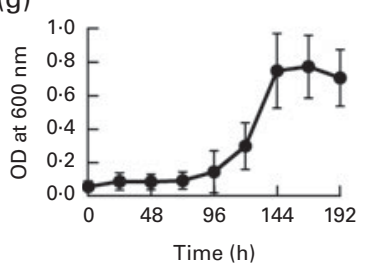

(i)

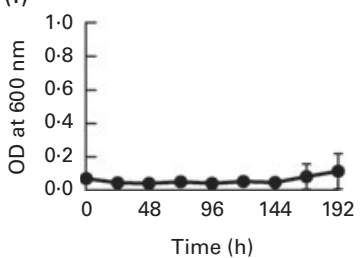

(b)

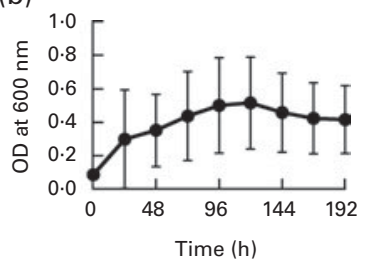

(d)

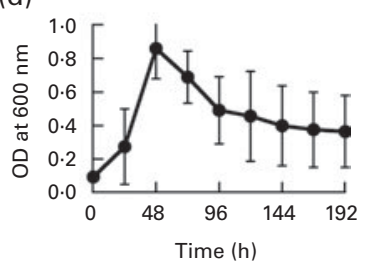

(f)

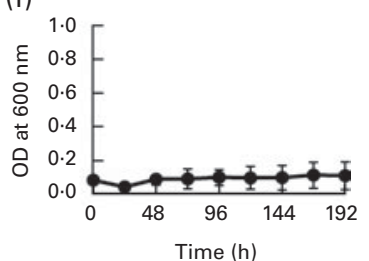

(h)

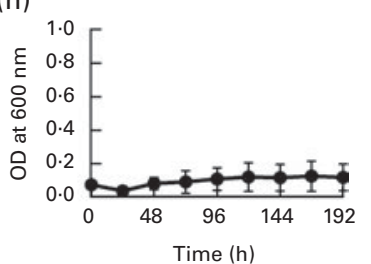

(j)

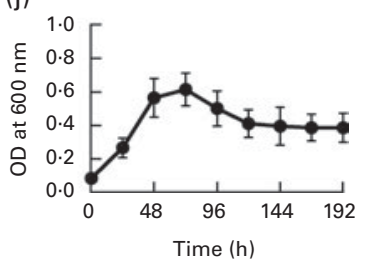

Fig. 4. Selective growth of Barnesiella intestinihominis (a, c, e, g, i) and $B$. viscericola (b, d, f, h, j) with different sugar substrates. The two Barnesiella strains were incubated in yeast extract, casitone, fatty acid medium supplemented with $(a, b)$ glucose, $(c, d)$ lactose, $(e, f)$ fucose, $(g, h)$ 2-fucosyllactose (2-FL) or (i, j) 3-fucosyllactose (3-FL). Proliferation was measured spectrophotometrically at $600 \mathrm{~nm}$. Values are means of two experiments carried out in triplicate, with standard deviations represented by vertical bars. OD, optical density.

Barnesiella was monitored by Gram staining to confirm the purity of the two strains in culture (Fig. S1, available online).

\section{Dextran sulphate sodium-induced colitis in the fucosyllactose-supplemented mice}

We finally investigated whether the changes in intestinal microbiota mediated by 2-FL and 3-FL supplementation affected the susceptibility of mice to local inflammation. Therefore, we treated mice with DSS to induce acute colitis. Loss of body weight was assessed to monitor the severity of the response to DSS in the different mouse groups.

Supplementation of 2-FL and 3-FL during lactation or for 3 weeks after weaning had no influence on the body-weight loss induced by DSS uptake (Fig. 5(a) and (b)). By contrast, 2-FL supplementation for 6 weeks decreased the severity of DSS-induced colitis (Fig. 5(c)). Similarly, long-term 3-FL supplementation led to reduced weight loss, although to a lesser extent than 2-FL supplementation. The difference between the supplementation groups was observed in male mice, which responded more strongly to DSS than females (Fig. 5(d)). Accordingly, male mice exposed to 2-FL and 3-FL maintained higher body weight values than the control mice, indicating a protective effect of the oligosaccharides towards exposure to DSS. The reduced severity of colitis observed in the 2-FL-supplemented mice was confirmed by measuring calprotectin concentrations in the faecal samples of treated mice (Fig. 5(e)). Calprotectin is secreted by infiltrating neutrophils and monocytes and is broadly used as a marker of intestinal inflammation ${ }^{(26,27)}$. The correlation of DSS sensitivity with the levels of Barnesiella bacteria in the intestine of the 2-FL-supplemented mice suggests that this bacterial genus may confer anti-inflammatory properties in the context of DSS challenge.

\section{Discussion}

The present study demonstrated that the exposure of young mice to the fucosyllactose isoforms 2-FL and 3-FL, which are absent in mouse milk, affects the composition of intestinal microbiota. Specifically, the levels of Barnesiella increased after 6 weeks of fucosyllactose supplementation. Barnesiella, a genus of the family of Porphyromonadaceae, order Bacteroidales, was one of the most abundant genera detected in mouse intestine. Already by 3 weeks of age, it accounted for up to $25 \%$ of total intestinal microbiota in mice. At the age of 6 weeks, this proportion increased to about $40 \%$ in the control mice, whereas the abundance of Barnesiella rose close to $60 \%$ in the 2-FL-supplemented mice. The dominance of these bacterial families in the murine gut was confirmed by Bailey et $a l .{ }^{(28)}$, who assigned approximately $30 \%$ of the identified sequences to each of these two families. Wohlgemuth et al. $^{(29)}$ also identified Porphyromonadaceae as the most abundant family with $37 \%$ of sequences, while they assigned $9 \%$ of sequences to Bacteroidaceae. Members of the order Bacteroidales contribute to carbohydrate degradation and by competition prevent the colonisation of the intestine by pathogenic bacteria ${ }^{(30)}$. Specifically, Barnesiella bacteria are able to eliminate and protect against the intestinal dominance of antibiotic-resistant pathogenic bacteria that can be observed in hospitalised patients ${ }^{(31)}$. The abundance of Barnesiella correlates with the amount of several immunoregulatory cells. The higher the levels of Barnesiella in the colon, the more marginal zone B cells and invariant natural killer T cells enumerated in the spleen and liver ${ }^{(32)}$. Furthermore, a direct association between a change in microbial composition in favour of Barnesiella and the resistance to arthritis has been demonstrated in mice ${ }^{(33)}$. Arthritis-resistant mice are characterised by microbiota enriched with Barnesiella, Bifidobacterium and Parabacteroides spp., with Barnesiella 

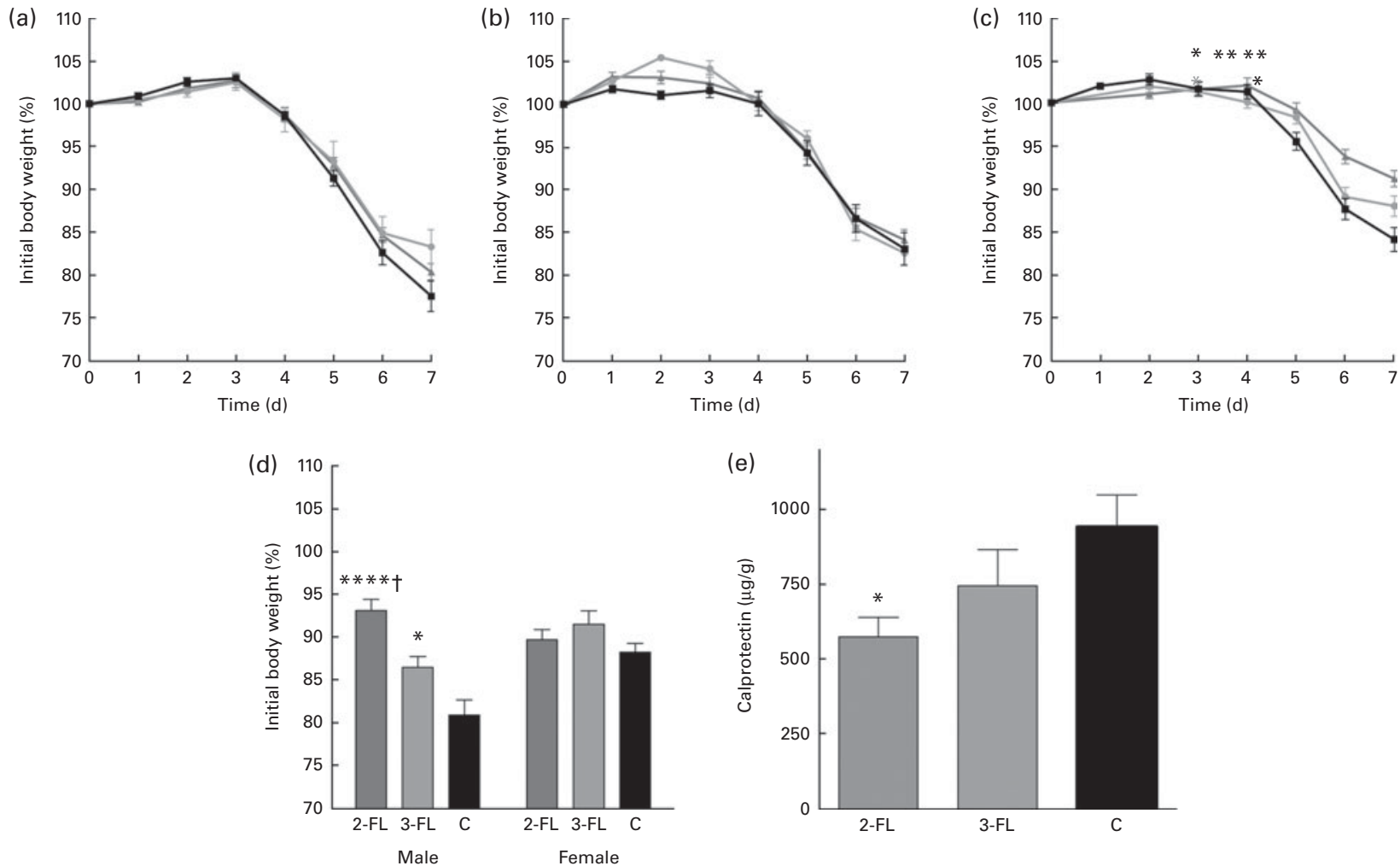

Fig. 5. Susceptibility of the fucosyllactose-supplemented mice to acute colitis. Acute colitis was induced in the fucosyllactose-supplemented and control mice by dextran sulphate sodium (DSS) treatment at the age of 6 weeks. Treatment with DSS in drinking-water was started on day 0 . On day 5 , treatment was ended by changing to normal drinking-water. Loss of body weight was monitored during DSS treatment and two subsequent days in mice supplemented for (a) 3 weeks until weaning, (b) 3 weeks after weaning and (c) 6 weeks (long-term supplementation). Values are means, with standard deviations represented by vertical bars. (a, c) Pooled data from two independent experiments. (a) Because of low body weight, two mice supplemented with 3-fucosyllactose (3-FL) for 3 weeks were killed by day 6 of DSS treatment. 2-FL - , 2-fucosyllactose ((a) $n$ 15, (b) $n 8$ and (c) $n 18) ; 3-F L-c-$ ((a) $n 12-14$, (b) $n 9$ and (c) $n 13)$; C - - , control ((a) $n 11$, (b) $n 8$ and (c) $n$ 16). Mean value was significantly different from that of the control mice: ${ }^{*} P<0.05,{ }^{* *} P<0.001$ ( $t$ test). (d) Loss of body weight in male and female mice was compared on day 7 after the start of DSS treatment in the long-term supplemented group. (e) Calprotectin concentrations in the faeces of the 2-FL- and 3-FL-supplemented mice (for 6 weeks) and control mice analysed on day 7 of DSS treatment. Values are means from pooled data of two independent experiments (males $n 8$ (2-FL), 9 (3-FL) and 9 (control) and females $n 10$ (2-FL), 4 (3-FL) and 7 (control)), with standard errors represented by vertical bars. Mean value was significantly different from that of the control mice: ${ }^{*} P<0.05$, ${ }^{* * \star} P<0.0001$ (one-way ANOVA). $†$ Mean value was significantly different from that of the 3-FL-supplemented mice $(P<0.05$; one-way ANOVA).

being the most abundant genus. In the development of colitis in $\mathrm{IL}-10^{-/-}$mice, higher levels of a Barnesiella phylotype correlated with lower activity levels of the disease ${ }^{(34)}$. In the last few years, many new sequences have been isolated from human and animal intestine and assigned to the genus Barnesiella $^{(35,36)}$. It still remains to be determined how the abundance of Barnesiella is linked to inflammatory diseases of the gastrointestinal tract.

As demonstrated in the present study, B. intestinibominis and $B$. viscericola can utilise fucosyllactose as energy source. This phenotype was contingent on the expression of linkagespecific fucosidases based on the respective specific utilisation of 2-FL and 3-FL by both Barnesiella species. B. intestinihominis had been previously tested negative ${ }^{(24)}$ and $B$. viscericola positive for $\alpha$-fucosidase ${ }^{(25)}$, although the linkage specificity of this activity was not addressed. The expression of glycosidases able to cleave carbohydrates capping lactose brings a selective advantage to bacteria competing for energy in the densely populated gastrointestinal environment. The gut of breast-fed newborns is enriched with Bacteroides and Bifidobacterium species that possess $\alpha$-fucosidase and $\alpha$-sialidase activity $^{(2,37-39)}$. As $\alpha$-fucosidase and $\alpha$-sialidase conduct the first step of milk oligosaccharide metabolisation by removing terminal monosaccharides, the expression of these two enzymes leads to the successful intestinal establishment of species such as Bifidobacterium infantis, Bifidobacterium bifidum, Bacteroides fragilis and Bacteroides thetaiotaomicron in breast-fed infants ${ }^{(37,40,41)}$. Bacteria that do not exhibit $\alpha$-fucosidase or $\alpha$-sialidase activity, e.g. Bifidobacterium adolescentis and Bifidobacterium animalis, are not able to grow solely on milk oligosaccharides and, therefore, do not belong to the typical infant-associated microbiota ${ }^{(42)}$. Little is known about the carbohydrate-metabolising activity of Barnesiella, but the feeding of obese mice with oligofructose has been shown to increase the abundance of Barnesiella by $26 \%{ }^{(43)}$.

As has been shown recently, specific milk oligosaccharides have a significant impact on the severity of intestinal inflammation $^{(9)}$. This effect seems to be microbiota mediated. The differential susceptibility of mice to DSS-induced colitis indicated that the shift in microbial composition elicited by 
2-FL supplementation had a significant biological impact on the health of the treated animals. The decreased susceptibility to DSS correlated with the levels of Barnesiella in the 2-FL-supplemented mice, suggesting that Barnesiella could render the intestinal milieu less prone to inflammation. The protective effect of 2-FL supplementation was strongest in male mice, whereas the long-term supplemented female mice did not differ significantly in their reaction to DSS from female control mice. Future experiments will examine whether the distribution of specific bacterial groups such as Barnesiella presents sex differences after fucosyllactose supplementation. Along this line, differences in the intestinal microbiome of male $v$. female mice were reported in the context of an arthritis susceptibility study, in which male mice had higher levels of $B$. viscericola than female mice ${ }^{(33)}$.

The present study demonstrated that supplementation with specific human milk oligosaccharides influenced the development of intestinal microbiota in mice as indicated by changes in the abundance of Barnesiella. Although Barnesiella bacteria belong to the major bacterial genera in mice, their representation in human microbiota is minor ${ }^{(30,44)}$ and changes in the composition of Barnesiella have not been reported yet to be associated with resistance to gastrointestinal disorders in humans. Nevertheless, supplementation of 2-FL might be of interest in children whose mothers lack 2-FL in breast milk. Approximately $20 \%$ of Caucasians have a genetic inability to express $\alpha(1,2)$-fucosyltransferase activity. In women, this results in the lack of $\alpha(1,2)$-linked fucose in milk oligosaccharides, thereby to 2 -FL deficiency in milk ${ }^{(10,45)}$. As $\alpha(1,2)$-fucosylated oligosaccharides inhibit the attachment of several enteric pathogens, the absence of these glycans is associated with different infectious diseases ${ }^{(14,46-48)}$. Current research focuses on the synthesis of fucosyllactose by Escherichia coli ${ }^{(49)}$ or with the help of bacterial enzymes ${ }^{(50,51)}$ for the production of food additives. To prevent disadvantages in children of the so-called non-secretors, especially in the context of necrotising enterocolitis, the supplementation of 2-FL might become a possible treatment option.

\section{Supplementary material}

To view supplementary material for this article, please visit http://dx.doi.org/10.1017/S0007114513004200

\section{Acknowledgements}

The authors cordially thank Glycom (Lyngby, Denmark) for providing 2-FL and 3-FL, the University Children's Hospital Zurich for enabling the DGGE analyses and Sacha Schneeberger for technical assistance.

The present study is part of a cooperative project funded by the University Research Priority Program 'Integrative Human Physiology' and by the Swiss National Foundation grant 31003A-116039 to T. H. The University Research Priority Program 'Integrative Human Physiology' and the Swiss National Foundation had no role in the design and analysis of the study or in the writing of this article.
The authors' contributions are as follows: G. A. W. and T. H. planned the study; G. A. W. carried out the experiments. All authors analysed and interpreted the data and wrote the manuscript.

The authors declare no conflicts of interest.

\section{References}

1. Ninonuevo MR, Park Y, Yin H, et al. (2006) A strategy for annotating the human milk glycome. J Agric Food Chem 54, 7471-7480.

2. Marcobal A \& Sonnenburg JL (2012) Human milk oligosaccharide consumption by intestinal microbiota. Clin Microbiol Infect 18, Suppl. 4, 12-15.

3. Kemp A \& Bjorksten B (2003) Immune deviation and the hygiene hypothesis: a review of the epidemiological evidence. Pediatr Allergy Immunol 14, 74-80.

4. Newburg DS (1996) Oligosaccharides and glycoconjugates in human milk: their role in host defense. J Mammary Gland Biol Neoplasia 1, 271-283.

5. Shoaf-Sweeney KD \& Hutkins RW (2009) Adherence, antiadherence, and oligosaccharides preventing pathogens from sticking to the host. Adv Food Nutr Res 55, 101-161.

6. Martin-Sosa S, Martin MJ \& Hueso P (2002) The sialylated fraction of milk oligosaccharides is partially responsible for binding to enterotoxigenic and uropathogenic Escherichia coli human strains. J Nutr 132, 3067-3072.

7. Velupillai P \& Harn DA (1994) Oligosaccharide-specific induction of interleukin 10 production by $\mathrm{B} 220^{+}$cells from schistosome-infected mice: a mechanism for regulation of $\mathrm{CD}^{+}{ }^{+}$T-cell subsets. Proc Natl Acad Sci U S A 91, 18-22.

8. Jantscher-Krenn E, Zherebtsov M, Nissan C, et al. (2012) The human milk oligosaccharide disialyllacto- $N$-tetraose prevents necrotising enterocolitis in neonatal rats. Gut 61, 1417-1425.

9. Fuhrer A, Sprenger N, Kurakevich E, et al. (2010) Milk sialyllactose influences colitis in mice through selective intestinal bacterial colonization. J Exp Med 207, 2843-2854.

10. Kunz C, Rudloff S, Baier W, et al. (2000) Oligosaccharides in human milk: structural, functional, and metabolic aspects. Annu Rev Nutr 20, 699-722.

11. Prieto PA, Mukerji P, Kelder B, et al. (1995) Remodeling of mouse milk glycoconjugates by transgenic expression of a human glycosyltransferase. J Biol Chem 270, 29515-29519.

12. Lowe JB (1993) The blood group-specific human glycosyltransferases. Baillieres Clin Haematol 6, 465-492.

13. De Leoz ML, Gaerlan SC, Strum JS, et al. (2012) Lacto- $N$ tetraose, fucosylation, and secretor status are highly variable in human milk oligosaccharides from women delivering preterm. J Proteome Res 11, 4662-4672.

14. Ruiz-Palacios GM, Cervantes LE, Ramos P, et al. (2003) Campylobacter jejuni binds intestinal $\mathrm{H}(\mathrm{O})$ antigen (Fuc $\alpha$ 1, $2 \mathrm{Gal} \beta 1$, 4GlcNAc), and fucosyloligosaccharides of human milk inhibit its binding and infection. J Biol Chem 278, 14112-14120.

15. Morrow AL, Ruiz-Palacios GM, Altaye M, et al. (2004) Human milk oligosaccharides are associated with protection against diarrhea in breast-fed infants. J Pediatr 145, 297-303.

16. Yu ZT, Chen C, Kling DE, et al. (2013) The principal fucosylated oligosaccharides of human milk exhibit prebiotic properties on cultured infant microbiota. Glycobiology 23, 169-177.

17. Hooper LV, Xu J, Falk PG, et al. (1999) A molecular sensor that allows a gut commensal to control its nutrient foundation in a competitive ecosystem. Proc Natl Acad Sci US A 96, 9833-9838. 
18. Wang Q, Garrity GM, Tiedje JM, et al. (2007) Naive Bayesian classifier for rapid assignment of rRNA sequences into the new bacterial taxonomy. Appl Environ Microbiol 73, 5261-5267.

19. Andersson AF, Lindberg $\mathrm{M}$, Jakobsson $\mathrm{H}$, et al. (2008) Comparative analysis of human gut microbiota by barcoded pyrosequencing. PLOS ONE 3, e2836.

20. Caporaso JG, Kuczynski J, Stombaugh J, et al. (2010) QIIME allows analysis of high-throughput community sequencing data. Nat Methods 7, 335-336.

21. DeSantis TZ, Hugenholtz P, Larsen N, et al. (2006) Greengenes, a chimera-checked 16S rRNA gene database and workbench compatible with ARB. Appl Environ Microbiol 72, 5069-5072.

22. Zhang Z, Schwartz S, Wagner L, et al. (2000) A greedy algorithm for aligning DNA sequences. J Comput Biol 7, 203-214.

23. Duncan SH, Hold GL, Harmsen HJ, et al. (2002) Growth requirements and fermentation products of Fusobacterium prausnitzii, and a proposal to reclassify it as Faecalibacterium prausnitzii gen. nov., comb. nov. Int J Syst Evol Microbiol 52 , 2141-2146.

24. Morotomi M, Nagai F, Sakon H, et al. (2008) Dialister succinatiphilus sp. nov. and Barnesiella intestinibominis sp. nov., isolated from human faeces. Int J Syst Evol Microbiol 58, 2716-2720.

25. Sakamoto M, Lan PT \& Benno Y (2007) Barnesiella viscericola gen. nov., sp. nov., a novel member of the family Porphyromonadaceae isolated from chicken caecum. Int J Syst Evol Microbiol 57, 342-346.

26. Tibble JA, Sigthorsson G, Bridger S, et al. (2000) Surrogate markers of intestinal inflammation are predictive of relapse in patients with inflammatory bowel disease. Gastroenterology 119, 15-22.

27. Carroll D, Corfield A, Spicer R, et al. (2003) Faecal calprotectin concentrations and diagnosis of necrotising enterocolitis. Lancet 361, 310-311.

28. Bailey MT, Dowd SE, Parry NM, et al. (2010) Stressor exposure disrupts commensal microbial populations in the intestines and leads to increased colonization by Citrobacter rodentium. Infect Immun 78, 1509-1519.

29. Wohlgemuth S, Keller S, Kertscher R, et al. (2011) Intestinal steroid profiles and microbiota composition in colitic mice. Gut Microbes 2, 159-166.

30. Kulagina EV, Efimov BA, Maximov PY, et al. (2012) Species composition of Bacteroidales order bacteria in the feces of healthy people of various ages. Biosci Biotechnol Biochem 76, 169-171.

31. Ubeda C, Bucci V, Caballero S, et al. (2013) Intestinal microbiota containing Barnesiella cures vancomycin-resistant Enterococcus faecium colonization. Infect Immun 81, 965-973.

32. Presley LL, Wei B, Braun J, et al. (2010) Bacteria associated with immunoregulatory cells in mice. Appl Environ Microbiol 76, 936-941.

33. Gomez A, Luckey D, Yeoman CJ, et al. (2012) Loss of sex and age driven differences in the gut microbiome characterize arthritis-susceptible 0401 mice but not arthritis-resistant 0402 mice. PLOS ONE 7, e36095.

34. Ye J, Lee JW, Presley LL, et al. (2008) Bacteria and bacterial rRNA genes associated with the development of colitis in IL-10 ${ }^{(-/-)}$mice. Inflamm Bowel Dis 14, 1041-1050.
35. Wylie KM, Truty RM, Sharpton TJ, et al. (2012) Novel bacterial taxa in the human microbiome. PLOS ONE 7, e35294.

36. Marti R, Zhang Y, Lapen DR, et al. (2011) Development and validation of a microbial source tracking marker for the detection of fecal pollution by muskrats. J Microbiol Methods 87, 82-88.

37. Marcobal A, Barboza M, Sonnenburg ED, et al. (2011) Bacteroides in the infant gut consume milk oligosaccharides via mucus-utilization pathways. Cell Host Microbe 10, 507-514.

38. Sela DA, Garrido D, Lerno L, et al. (2012) Bifidobacterium longum subsp. infantis ATCC $15697 \alpha$-fucosidases are active on fucosylated human milk oligosaccharides. Appl Environ Microbiol 78, 795-803.

39. Reuter G \& Gabius HJ (1996) Sialic acids structure-analysismetabolism-occurrence-recognition. Biol Chem Hoppe Seyler 377, 325-342.

40. Turroni F, Bottacini F, Foroni E, et al. (2010) Genome analysis of Bifidobacterium bifidum PRL2010 reveals metabolic pathways for host-derived glycan foraging. Proc Natl Acad Sci U S A 107, 19514-19519.

41. Sela DA, Chapman J, Adeuya A, et al. (2008) The genome sequence of Bifidobacterium longum subsp. infantis reveals adaptations for milk utilization within the infant microbiome. Proc Natl Acad Sci U S A 105, 18964-18969.

42. Chichlowski M, German JB, Lebrilla CB, et al. (2011) The influence of milk oligosaccharides on microbiota of infants: opportunities for formulas. Annu Rev Food Sci Technol 2, $331-351$.

43. Everard A, Lazarevic V, Derrien M, et al. (2011) Responses of gut microbiota and glucose and lipid metabolism to prebiotics in genetic obese and diet-induced leptin-resistant mice. Diabetes 60, 2775-2786.

44. Shkoporov AN, Khokhlova EV, Kulagina EV, et al. (2008) Application of several molecular techniques to study numerically predominant Bifidobacterium spp. and Bacteroidales order strains in the feces of healthy children. Biosci Biotechnol Biochem 72, 742-748.

45. Grollman EF \& Ginsburg V (1967) Correlation between secretor status and the occurrence of $2^{\prime}$-fucosyllactose in human milk. Biochem Biophys Res Commun 28, 50-53.

46. Le Pendu J (2004) Histo-blood group antigen and human milk oligosaccharides: genetic polymorphism and risk of infectious diseases. Adv Exp Med Biol 554, 135-143.

47. Newburg DS, Ruiz-Palacios GM \& Morrow AL (2005) Human milk glycans protect infants against enteric pathogens. Annu Rev Nutr 25, 37-58.

48. Morrow AL, Ruiz-Palacios GM, Jiang X, et al. (2005) Humanmilk glycans that inhibit pathogen binding protect breastfeeding infants against infectious diarrhea. J Nutr 135, 1304-1307.

49. Lee WH, Pathanibul P, Quarterman J, et al. (2012) Whole cell biosynthesis of a functional oligosaccharide, $2^{\prime}$-fucosyllactose, using engineered Escherichia coli. Microb Cell Fact 11, 48

50. Albermann C, Piepersberg W \& Wehmeier UF (2001) Synthesis of the milk oligosaccharide 2 -fucosyllactose using recombinant bacterial enzymes. Carbohydr Res 334, 97-103.

51. Murata T, Morimoto S, Zeng X, et al. (1999) Enzymatic synthesis of $\alpha$-L-fucosyl- $N$-acetyllactosamines and $3^{\prime}-O$-alpha-Lfucosyllactose utilizing $\alpha$-L-fucosidases. Carbohydr Res 320, 192-199. 